INPLASY

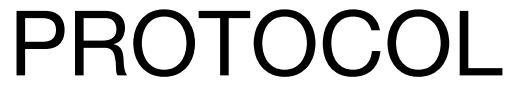

To cite: Liu et al. Meta-analysis of the serum galectin- 3 in heart failure. Inplasy protocol 202150013. doi:

10.37766/inplasy2021.5.0013

Received: 03 May 2021

Published: 03 May 2021

Corresponding author: Qin Wang

Wangq@swmu.edu.cn

Author Affiliation:

Affiliated Hospital of

Southwest Medical University

Support: Southwest Medical University.

Review Stage at time of this submission: Data analysis.

Conflicts of interest:

None declared.

\section{Meta-analysis of the serum galectin-3 in heart failure}

Liu, XQ1; Ye, WX2; Han,YS3; Fu, W4; Tong, R5; Wang, Q6.

Review question / Objective: Quantify the association between serum galectin-3 levels and the presence of heart failure. Therefore, we conducted a meta-analysis of the available clinical studies on this topic.

Condition being studied: Currently, in HF patients, an increase in serum levels of several substances has been observed, such as natriuretic peptides, cytokines, and tumor markers, and their use has been proposed in diagnosis, prognostic stratification, and therapeutic monitoring of this common disease. Of interest, among several tumor markers evaluated, only variations in serum galectin-3 (Gal-3) seem to be clinically related to heart failure. In the last decade, several studies have been published showing an increase of Gal-3 serum levels in patients with symptomatic left ventricular dysfunction. However, the potential role of Gal-3 in the management of patients with suspected or established heart failure has not been clarified yet. Accordingly, in order to quantify the association between Gal-3 levels and the presence of heart failure, we conducted the meta-analysis of the available clinical studies on this topic.

INPLASY registration number: This protocol was registered with the International Platform of Registered Systematic Review and Meta-Analysis Protocols (INPLASY) on 03 May 2021 and was last updated on 03 May 2021 (registration number INPLASY202150013).

\section{INTRODUCTION}

Review question / Objective: Quantify the association between serum galectin-3 levels and the presence of heart failure. Therefore, we conducted a meta-analysis of the available clinical studies on this topic.

Condition being studied: Currently, in HF patients, an increase in serum levels of several substances has been observed, such as natriuretic peptides, cytokines, and 
tumor markers, and their use has been proposed in diagnosis, prognostic stratification, and therapeutic monitoring of this common disease. Of interest, among several tumor markers evaluated, only variations in serum galectin-3 (Gal-3) seem to be clinically related to heart failure. In the last decade, several studies have been published showing an increase of Gal-3 serum levels in patients with symptomatic left ventricular dysfunction. However, the potential role of Gal-3 in the management of patients with suspected or established heart failure has not been clarified yet. Accordingly, in order to quantify the association between Gal-3 levels and the presence of heart failure, we conducted the meta-analysis of the available clinical studies on this topic.

\section{METHODS}

Participant or population: A total of 2042 HF patients and 939 healthy controls (HCs) from 18 studies were included in the analysis.

Intervention: None.

Comparator: None.

Study designs to be included: A comprehensive literature search was conducted by searching the PubMed, EMBASE, Web of Science, Cochrane, the Chinese National Knowledge Infrastructure database (CNKI), and the Wanfang online databases from inception to September 1, 2020. We used the standard mean difference (SMD) with a $95 \%$ confidence interval $(\mathrm{Cl})$ to estimate the pooled effect and used subgroup analysis to investigate heterogeneity.

Eligibility criteria: This meta-analysis is compliant with the Preferred Reporting Items for Systematic Reviews and MetaAnalyses (PRISMA) statement.

Information sources: A comprehensive literature search was conducted by searching the PubMed, EMBASE, Web of Science, Cochrane, the Chinese National
Knowledge Infrastructure database (CNKI), and the Wanfang online databases.

Main outcome(s): A total of 2042 HF patients and 939 healthy controls (HCs) from 18 studies were included in the analysis. The Gal-3 value was significantly higher in 2042 HF patients than that in 939 HCs $(S M D=1.26,95 \% C I=0.97-1.55$, $P<0.001)$; in 578 SHF patients than that in $320 \mathrm{HCs}(\mathrm{SMD}=1.4,95 \% \mathrm{Cl}=0.96-1.84$, $P<0.001)$; and in 516 DHF patients than that in $208 \mathrm{HCs}(\mathrm{SMD}=1.41,95 \% \mathrm{Cl}=0.89-1.98$, $P<0.001)$. The Gal-3 was no difference in 444 DHF patients than that in 825 SHF patients (SMD $=-0.2,95 \% \mathrm{Cl}=-0.89-$ $0.31, P<0.001)$.

Quality assessment / Risk of bias analysis: The funnel plot was asymmetric of Gal-3 between heart failure and healthy controls, and the P-value was $<0.001$ in Begg's test and 0.049 in Egger's test, thus indicating a potential publication bias.

Strategy of data synthesis: All statistical analyses were performed using STATA software version 14.0 (STATA Corp., College Station, TX, USA). Weighted mean difference (WMD) values were reported with $95 \%$ confidence intervals (Cls), and Pvalue $<0.05$ was considered statistically significant. Data presented as a median with IQR were finally converted to mean with SD using a previously described formula. We used the $Q$ statistic to test the heterogeneity of studies (the significance level at $P<0.10$ ). The heterogeneity (12) statistic was also calculated $(12<25 \%$, no heterogeneity; 12 between $25 \%$ and $50 \%$, moderate heterogeneity; 12 between $50 \%$ and $75 \%$, large heterogeneity; and $12>$ $75 \%$, extreme heterogeneity). Due to the relatively high heterogeneity within studies, we used a random-effect model to calculate the pooled WMD and $95 \% \mathrm{Cls}$. Besides, we used Begg's adjusted rank correlation test and Egger's regression asymmetry test to detect publication bias. $P>0.05$ for both tests was considered to be no significant publication bias. We conducted a sensitivity analysis to investigate an individual study's influence on overall risk estimate by sequentially 
excluding one study in each step. The Duval and Tweedie "trim and fill" procedure was performed to assess the possible effect of publication bias further.

Subgroup analysis: The Gal-3 in SHF patients were higher than those in $\mathrm{HCs}$, the gAL-3 DHF patients were higher than those in HCs.

Sensitivity analysis: The sensitivity analysis of Gal-3 between HF and HCs showed that the pooled SMD did not materially change when each study was sequentially excluded.

Country(ies) involved: China.

Keywords: Galectin-3; Heart failure;

Biomarker; Meta-analysis.

Contributions of each author:

Author 1 - Xiangqiang Liu.

Author 2 - Weixia Ye.

Author 3 - Yinshuo Han.

Email: 1165767817@qq.com

Author 4 - Wei Fu.

Author 5 - Ring Tang.

Author 6 - Qin Wang.

Email: Wangq@swmu.edu.cn 